R.E. Baker · S. Schnell · P.K. Maini

\title{
A mathematical investigation of a Clock and Wavefront model for somitogenesis
}

Received: 18 May 2005 / Revised version: 16 October 2005 /

Published online: 7 February 2006 - (C) Springer-Verlag 2006

\begin{abstract}
Somites are transient blocks of cells that form sequentially along the antero-posterior axis of vertebrate embryos. They give rise to the vertebrae, ribs and other associated features of the trunk. In this work we develop and analyse a mathematical formulation of a version of the Clock and Wavefront model for somite formation, where the clock controls when the boundaries of the somites form and the wavefront determines where they form. Our analysis indicates that this interaction between a segmentation clock and a wavefront can explain the periodic pattern of somites observed in normal embryos. We can also show that a simplification of the model provides a mechanism for predicting the anomalies resulting from perturbation of the wavefront.
\end{abstract}

\section{Introduction}

There is increasing experimental evidence to suggest that the graded expression of certain morphogens is vital to the generation of structure within a developing embryo. Somitogenesis is one example of a developmental process which utilises this process as a control mechanism.

Somites are tightly bound blocks of cells that lie along the antero-posterior (AP) axis of vertebrate embryos; they are transient structures and further differentiation of the somites gives rise to the vertebrae, ribs and other associated features of the trunk [8]. Somitogenesis is tightly regulated in both space and time [11], with each somite forming from a seemingly uniform field of cells via a mechanism that involves the interaction of a moving gradient of morphogen and a segmentation clock. Somitogenesis is one of the most well studied examples of pattern formation

R.E. Baker: Centre for Mathematical Biology, Mathematical Institute, 24-29 St Giles', Oxford, OX1 3LB, UK. e-mail: ruth. baker@maths. ox. ac.uk

S. Schnell: Christ Church, Oxford, OX1 1DP, UK.

Centre for Mathematical Biology, Mathematical Institute, 24-29 St Giles', Oxford, OX1 3LB, UK. e-mail: schnell@maths.ox.ac.uk

Present address: Indiana School of Informatics and Biocomplexity Institute, Eigemann Hall 906, 1900 East 10th Street, Bloomington, Indiana 47406, USA.

e-mail: schnell@indiana. edu

P.K. Maini: Centre for Mathematical Biology, Mathematical Institute, 24-29 St Giles', Oxford, OX1 3LB, UK. e-mail: maini@maths.ox.ac.uk

Key words or phrases: Somitogenesis - Clock and Wavefront model - Segmentation clock FGF8 wavefront - Travelling waves 
in the developing embryo and is becoming, more and more, a leading candidate in developmental biology for a study that aims to couple findings at a molecular level with those at a cell and tissue level and lends itself openly to investigation from a more theoretical viewpoint [19].

Events leading to the formation of somites occur as follows: two parallel bands of cells, known collectively as the pre-somitic mesoderm (PSM), lie alongside the notochord (the precursor of the spinal chord). At regular time intervals (every 90 minutes in the chick), groups of cells at the very anterior end of each strip of the PSM undergo changes in their adhesive and migratory properties and coalesce to form an epithelial block of cells known as a somite. In this way, somites form in a very strict AP sequence $[8,21,22]$ and the budding of cells from the anterior end of the PSM compensates for the addition of cells at the posterior end of the PSM as the body axis lengthens. In this way, each band of the PSM stays approximately constant in length throughout the process of segmentation and a wave of cell determination appears to sweep along the AP axis leaving somites in its wake [18,19].

The Clock and Wavefront model for somitogenesis was first postulated by Cooke and Zeeman [4]: a longitudinal positional information gradient was proposed to exist along the AP axis of the embryo, determining regional development by interacting with a segmentation clock to set the time in each cell at which it undergoes a catastrophe. By catastrophe, they meant a rapid change of state, which could possibly be the change in the adhesive behaviour of cells when they become part of a somite.

Discovery of the periodic expression of c-hairy- 1 and several other genes related to the Notch signalling pathway provides a molecular basis for the existence of a segmentation clock [10]. Recent experimental evidence concerning the existence of a moving gradient of FGF8 expression along the AP axis of vertebrate embryos [5] has provided a basis for the wavefront and an experimentally grounded version of the Clock and Wavefront model has recently been proposed by Pourquié and co-workers $[6,12]$.

\subsection{Aims and outline}

In previous papers we have analysed the effects of heat shock on somite formation [3,9], included cell movement during the segmentation process [18] and analysed the effects of local application or inhibition of FGF8 on somitogenesis [1].

Here we develop and analyse a mathematical formulation of the Clock and Wavefront model proposed by Pourquié and co-workers [5,6]. Our model is an extension of a mathematical formulation proposed by Collier et al. [3] consisting of two coupled partial differential equations, whose non-linear coupling gives rise to a regular pulse-like signal. The new mathematical model introduces two additional partial differential equations which incorporate the wavefront responsible for the formation of somites.

In Section 2 we detail a word model and accompanying mathematical formulation based on the previous models of Maini and co-workers [1,3,9,19]. We find analytical solutions for the FGF8 profile along the AP axis and make some simplifying assumptions (Section 4). We solve the simplified model numerically and present an extension to include the effects of local application of FGF8, with 


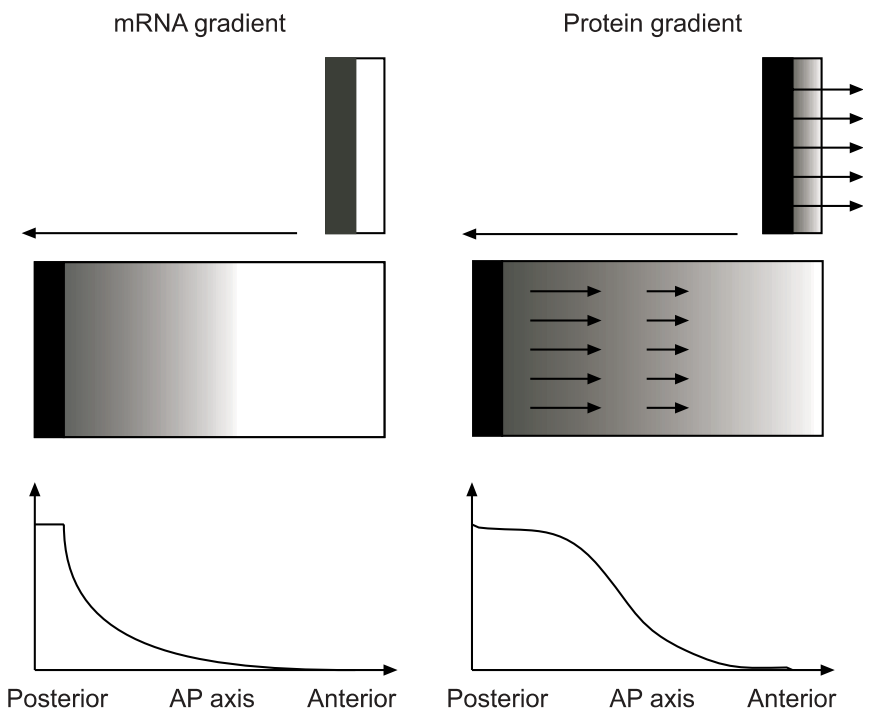

Fig. 1. An illustration of the manner in which the gradient of FGF8 is maintained along the AP axis. As the tail bud regresses and creates the PSM, cells entering the PSM are endowed with a base level of $f g f 8$ which degrades over time (see mRNA gradient). Local production of FGF8 by such cells creates a more shallow protein gradient (see Protein gradient).

both a schematic view of the resulting anomalies and numerical simulation of the mathematical model (Section 5). This is followed by a brief discussion (Section 6).

\section{Formulation of the model}

The work of Pourquié and co-workers, amongst others, over the past decade has provided an experimental basis for the theoretical Clock and Wavefront model first put forward by Cooke and Zeeman [4]. Pourquié and co-workers' [5,6] experimentally grounded version of the model hypothesises that there is some interaction between the moving wavefront of FGF8 and the segmentation clock that acts to gate cells into somites. For a cell at a particular point, they assume that competence to segment is only achieved once FGF8 signalling has decreased below a certain threshold, the position of which is known as the determination front [5].

Progression of the FGF8 wavefront along the AP axis of the embryo occurs as the body axis lengthens. New cells become part of the posterior PSM and they are assumed to transcribe $f g f 8$ at a constant rate. Upon leaving the tail of the embryo these cells lose their ability to transcribe $f g f 8$ and their levels decrease as $f g f 8$ undergoes linear decay. FGF8 is translated by cells expressing $f g f 8$ and the FGF8 diffuses along the AP axis to create a signalling gradient. Figure 1 shows the manner in which the FGF8 gradient is established and maintained. As illustrated in the figure, high levels of FGF8 signalling prevail in the posterior PSM and signalling levels decrease with movement in the anterior direction. In this way cells experience lessening levels of FGF8 signalling as they move up through the PSM [6]. 


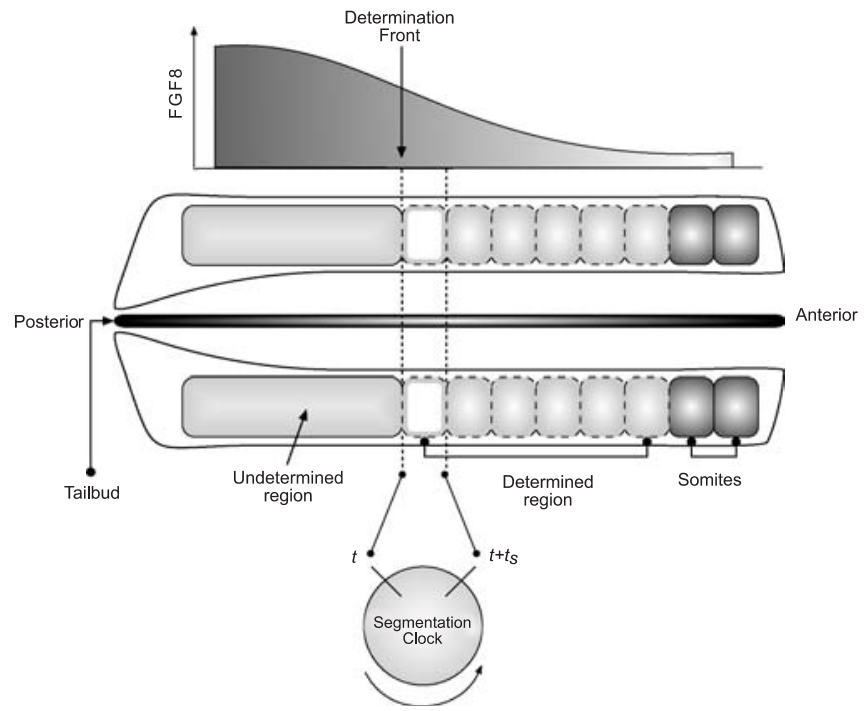

Fig. 2. Diagrammatic representation of the vertebrate body plan during somite formation. In the top part of the diagram the FGF8 wavefront is illustrated together with the position of the determination front. The middle section of the diagram shows the AP axis of the embryo with the somites (dark grey blocks), determined region (light grey blocks) and the undetermined region (light grey band) clearly marked. The bottom part of the diagram shows the segmentation clock with the time $t$ at which cells reach the determination front and the time $t_{s}$ later at which they become competent to signal. The hollow grey block marks the position of the next somite to be specified: the posterior boundary is fixed by the position of the determination front at the time at which cells at the anterior boundary become able to signal.

There are differences in both the structure of the PSM and the degree of segmental determination between the region of high FGF8 expression (posterior PSM) and the region of low FGF8 expression (anterior PSM). Cells in the posterior PSM are arranged in a loose mesenchymal manner and are labile with respect to their response to signalling (undetermined) whereas in the anterior parts of the PSM the cell arrangement has become more compact and the epithelialisation process has begun (determined) [5].

The border which separates the two regions of FGF8 signalling is the determination front (mentioned above) and it has been shown that cells require a level of FGF8 signalling below that expressed at the determination front in order to be capable of segmentation [5]. Figure 2 illustrates the vertebrate body plan during somite formation with the determination front clearly marked.

To develop a mathematical formulation of Pourquié's Clock and Wavefront model, we constructed a system of equations in line with the word model, in which the clock controls when the boundaries of the somites form and the wavefront determines where they form. This is in agreement with Pourquié and co-workers' observations [5,23]. In addition, we introduce the following assumptions:

- Once cells have reached the determination front they become competent to segment by gaining the ability to respond to a chemical signal, thereby producing a somitic factor. 
- A certain time $t_{s}$ after reaching the determination front cells gain the ability to produce the aforementioned signal. $t_{s}$ is equal to the period of the segmentation clock, which is coincident with the period of the cycling genes.

- Once a cell has reached the determination front and become part of a somite it becomes refractory to FGF8 signalling.

The model is based on the signalling model for somitogenesis presented by Maini and co-workers $[1,3,9,19]$ : at a certain time, a small fraction of cells at the anterior-most end of the PSM will have undergone a whole oscillation of the segmentation clock after reaching the determination front. These pioneer cells will produce and emit a signal which will diffuse along the PSM. Any cell which has a level of FGF8 below that expressed at the determination front will respond to the signal by increasing its adhesion to neighbouring cells which are behaving in a similar manner, thereby forming a potential somite. At this point, a cell is specified as somitic and it will go on to form a somite during subsequent oscillations of the segmentation clock. The process begins once again when cells now at the anterior end of the PSM become competent to signal. Emission of the signal is transient due to negative feedback on signal production by cells which are competent to react to the signal. This feedback loop results in periodic pulses in the signal and hence the specification of somites at regular time intervals. Figure 2 illustrates the vertebrate body plan during somitogenesis; the interaction of the clock and the wavefront is clearly shown.

Here we develop a mathematical formulation of Pourquié's descriptive Clock and Wavefront model consisting of a coupled system of four non-linear partial differential equations in time and one spatial dimension (the AP axis). The state variables which the system describes are a somitic factor which determines the fate of cells (only cells with a high level of somitic factor will go on to form part of a somite), a diffusive signalling molecule which is produced by pioneer cells at the anterior-most end of the PSM, fgf8 mRNA ( $f g f 8)$, and finally fgf8 protein (FGF8). We propose the following model for somite formation:

$$
\begin{aligned}
\frac{\partial u}{\partial t} & =\frac{(u+\mu v)^{2}}{\gamma+\rho u^{2}} \chi_{u}-v u, \\
\frac{\partial v}{\partial t} & =\frac{\kappa}{\epsilon+u} \chi_{v}-\lambda v+D_{v} \frac{\partial^{2} v}{\partial x^{2}}, \\
\frac{\partial m}{\partial t} & =\Lambda \chi_{m}-\beta m, \\
\frac{\partial p}{\partial t} & =\Gamma m-\eta p+D_{p} \frac{\partial^{2} p}{\partial x^{2}},
\end{aligned}
$$

where

$$
\begin{aligned}
\chi_{u}(x, t) & =H\left(p^{*}-p\right), \\
\chi_{v}(x, t) & =H\left(t-t^{*}(x)-t_{s}\right), \\
\chi_{m}(x, t) & =H\left(x-x_{n}-c_{n} t\right) .
\end{aligned}
$$


$u$ is the concentration of somitic factor, $v$ is the concentration of signalling molecule and $m$ and $p$ are the concentrations of fgf 8 mRNA and protein, respectively. $\mu, \gamma$, $\rho, v, \kappa, \epsilon, \lambda, \Lambda, \beta, \Gamma, \eta, t_{s}, x_{n}, c_{n}, D_{v}, D_{p}$ and $p^{*}$ are positive constants and

$$
p\left(x, t^{*}(x)\right)=p^{*},
$$

i.e. $t^{*}(x)$ describes the path of the determination front in the $(x, t)$ plane.

Somitic factor production is activated by the signal and is self-regulating as levels become high. High levels of somitic factor also inhibit production of the signal, which is able to diffuse. For a more detailed explanation of the system of equations describing the somitic factor and the signal see $[3,9,19]$. $f g f 8$ is produced only in the tail region of the embryo which is assumed to be initially at $x_{n}$ and the axis is assumed to extend with speed $c_{n}$. In this way the regression speed of the wavefront is coupled to the speed of axis elongation: this has been shown experimentally by Pourquié and co-workers [7]. $f g f 8$ translates FGF8 and FGF8 diffuses along the AP axis. All substances are assumed to undergo linear decay.

\subsection{Non-dimensionalisation}

We non-dimensionalise by taking

$$
\begin{aligned}
t & =\frac{\hat{t}}{v}, \quad x=\bar{x} \hat{x}, \quad m=\frac{\Lambda}{v} \hat{m}, \quad p=\frac{\Lambda \Gamma}{\beta v^{2}} \hat{p}, \\
\beta & =\frac{\hat{\beta}}{v}, \quad \eta=\frac{\hat{\eta}}{v}, \quad p^{*}=\frac{\Lambda \Gamma}{\beta v^{2}} \hat{p}^{*}, \quad t^{*}=\frac{t^{*}}{v}, \\
t_{s} & =\frac{\hat{t_{s}}}{v}, \quad D_{p}=v \bar{x}^{2} \hat{D}_{p}, \quad x_{n}=\bar{x} \hat{x}_{n}, \quad c_{n}=v \bar{x} \hat{c}_{n},
\end{aligned}
$$

where $\bar{x}$ is the length of a somite. Dropping the hats for notational convenience, the system of non-dimensionalised equations becomes

$$
\begin{aligned}
\frac{\partial u}{\partial t} & =\frac{(u+\mu v)^{2}}{\gamma+u^{2}} \chi_{u}-u, \\
\frac{\partial v}{\partial t} & =\kappa\left(\frac{\chi_{v}}{\epsilon+u}-v\right)+D_{v} \frac{\partial^{2} v}{\partial x^{2}}, \\
\frac{\partial m}{\partial t} & =\chi_{m}-\beta m, \\
\frac{\partial p}{\partial t} & =\beta m-\eta p+D_{p} \frac{\partial^{2} p}{\partial x^{2}},
\end{aligned}
$$

with $\chi_{u}, \chi_{v}$ and $\chi_{m}$ as before.

\subsection{Boundary conditions}

We take boundary conditions for $u$ and $v$ similar to those used in [9]. Cells which are a long way from reaching the determination front have both the level of somitic factor and signalling molecule close to zero, while cells which reached the 
determination front some time ago have some fixed level of the somitic factor and signalling molecule.

For the fgf 8 concentrations, we assume that cells which reached the determination front some time ago have $f g f 8$ and FGF8 levels close to zero and that cells which are a long way from the determination front have fixed levels of $f g f 8$ and FGF8. We assume that the position of the determination front is close to the midpoint of the FGF8 profile and therefore lies at approximately $x=x_{n}+c_{n} t$. Hence we take our boundary conditions to be

$$
\begin{aligned}
& u, v \rightarrow 0 \text { as } x-\left\{x_{n}+c_{n} t\right\} \rightarrow+\infty \\
& u, v \text { are bounded as } x-\left\{x_{n}+c_{n} t\right\} \rightarrow-\infty \\
& m, p \text { are bounded as } x-\left\{x_{n}+c_{n} t\right\} \rightarrow+\infty \\
& m, p \rightarrow 0 \text { as } x-\left\{x_{n}+c_{n} t\right\} \rightarrow-\infty
\end{aligned}
$$

\subsection{Initial conditions}

We take the initial conditions for $u$ and $v$ to be as in [9]:

$$
u(x, 0)=\left\{\begin{array}{l}
1 \text { if } x \leq 0 \\
0 \text { if } x>0
\end{array}\right.
$$

and

$$
v(x, 0)=A^{*} H(-x)+B^{*} \cosh (\lambda(l-|x|)),
$$

where

$$
A^{*}=\frac{1}{1+\epsilon-\epsilon_{1}}, \quad B^{*}=\frac{A^{*} \operatorname{sign}(x)}{2 \cosh (\lambda l)}, \quad \lambda=\sqrt{\frac{\kappa}{D_{v}}},
$$

and $\epsilon_{1} \ll 1$.

The initial conditions for $m$ and $p$ are based on the travelling wave solutions of equations (14) and (15) at $t=0$ (see Section 3.1 and Section 3.2 for more details) and are such that:

$$
m(x, 0)= \begin{cases}\frac{1}{\beta} \exp \left[\beta\left(\frac{x-x_{n}}{c_{n}}\right)\right] & \text { if } \quad x \leq x_{n} \\ \frac{1}{\beta} & \text { if } \quad x>x_{n}\end{cases}
$$

and

$$
p(x, 0)=\left\{\begin{array}{lll}
A_{l} \exp \left\{n_{+}\left(x-x_{n}\right)\right\}+A \exp \left\{\beta \frac{\left(x-x_{n}\right)}{c_{n}}\right\} & \text { if } & x-x_{n} \leq 0, \\
B_{r} \exp \left\{n_{-}\left(x-x_{n}\right)\right\}+\frac{1}{\eta} & \text { if } & x-x_{n}>0,
\end{array}\right.
$$

where $A, n_{ \pm}, A_{l}$ and $B_{r}$ are given by

$$
A=\frac{1}{\left(\eta-\beta-\frac{D_{p} \beta^{2}}{c_{n}^{2}}\right)}, \quad n_{ \pm}=\frac{-c_{n} \pm \sqrt{c_{n}^{2}+4 \eta D_{p}}}{2 D_{p}}
$$


and

$$
A_{l}=\frac{1}{n_{+}-n_{-}}\left(n_{-} A-\frac{n_{-}}{\eta}-\frac{\beta A}{c_{n}}\right), \quad B_{r}=\frac{1}{n_{+}-n_{-}}\left(n_{+} A-\frac{n_{+}}{\eta}-\frac{\beta A}{c_{n}}\right) .
$$

Note that these conditions assume that the last formed somite occupies a region up to $x=0$ and so we must choose the position of the determination front accordingly to ensure that the next somite forms as a result of a signal produced by cells at $x=0$.

\subsection{Numerical solution of the model}

We solved the system of equations given by (12)-(15) numerically using the NAG library routine D03PCF, which is designed for non-linear parabolic (including some elliptic) partial differential equations in one spatial variable (see [2] for more details). We used zero flux boundary conditions on a closed bounded interval to approximate the infinite domain boundary conditions and continuous tanh functions to approximate the Heaviside functions.

The parameter values for the $u, v$ sub-system of equations are taken to ensure that the model is in a parameter regime which gives rise to coherent somites. This has been investigated in detail by the authors in a previous paper [9] and so we do not discuss it here. The remaining parameters for the fgf 8 mRNA and protein equations can be chosen to match the speed of the wavefront and the observed levels of fgf8 in the PSM. However, at present, there is no quantitative data available and so we have estimated these remaining parameters.

The level of FGF8 at the determination front is taken so that cells at the anterior edge of the PSM (i.e. cells at $x=0$ given the initial conditions stated previously) are able to signal at $t=0$. That is, they reached the determination front a time $t=t_{s}$ previously.

Figure 3 shows the numerical solution of the model for a control embryo. The figure shows a sequence of coherent jumps in the somitic factor as a result of a set of periodic pulses in the signalling molecule. The numerical solution is in agreement with experimental observations.

\section{Analysis of the mathematical model}

Although the results from numerical simulation of the model are promising, there is little experimental data available on any of the model parameters and therefore further analytic study is required to make sure that the numerical results are representative of the general model behaviour. Extensive phase plane analysis of the signalling basis of the model ( $u$ and $v$ equations) has been presented by McInerney et al. in [9]. Here we present some analytical results for the decoupled equations describing the fgf8 mRNA and protein dynamics ( $m$ and $p$ ). 
(a) Somitic factor (u)

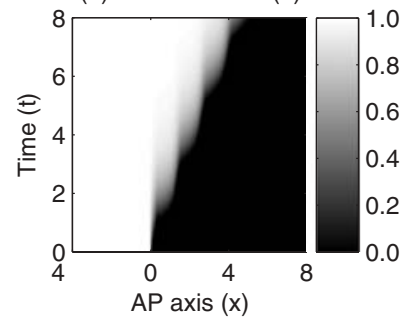

(c) fgf8 mRNA (m)

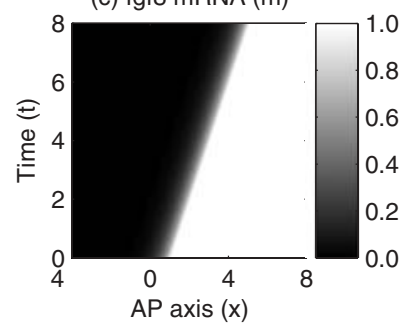

(b) Signal (v)

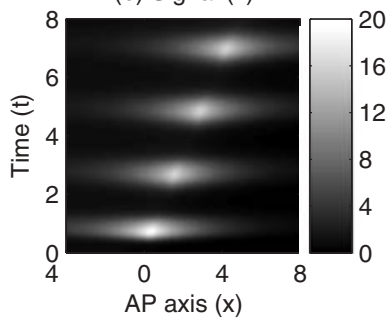

(d) fgf8 protein (p)

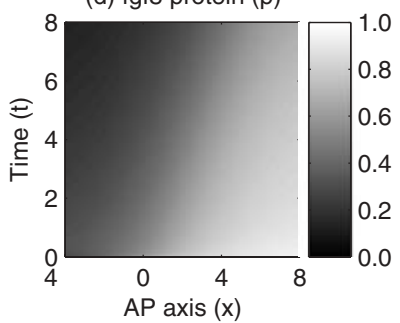

Fig. 3. Numerical solution of the new mathematical formulation of the Clock and Wavefront model for somite formation, given by equations (12)-(15), showing the spatio-temporal dynamics of the somitic factor (a), the signalling molecule, (b), fgf8, (c), and FGF8, (d). Parameters are as follows: $\mu=10^{-4}, \gamma=10^{-3}, \kappa=10, \epsilon=10^{-3}, \epsilon_{1}=10^{-3}, \eta=1.0$, $D_{v}=50, D_{p}=20, x_{n}=0.0, c_{n}=0.5$.

\subsection{The $m R N A$ profile}

Equation (14) for the mRNA profile can be solved to give

$$
m(x, t)= \begin{cases}m(x, 0) e^{-\beta t} & \text { if } \quad x \leq x_{n} \\ m(x, 0) e^{-\beta t}+\frac{1}{\beta}\left[e^{\beta \Sigma(x, t)}-1\right] e^{-\beta t} & \text { if } \quad x>x_{n}\end{cases}
$$

where $\Sigma=\min \left\{t,\left(x-x_{n}\right) / c_{n}\right\}$.

This study does not consider the initial stages of somitogenesis and we are therefore only interested in the $f g f 8$ gradient once levels have reached a steady state profile relative to the PSM. For this it is sufficient to take the initial conditions to be

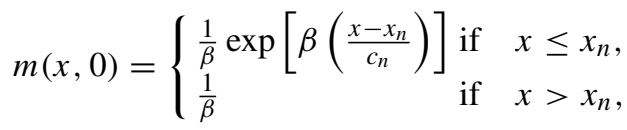

giving the result

$$
m(x, t)= \begin{cases}\frac{1}{\beta} \exp \left[\beta\left(\frac{x-x_{n}}{c_{n}}-t\right)\right] & \text { if } \quad x \leq x_{n}+c_{n} t \\ \frac{1}{\beta} & \text { if } \quad x>x_{n}+c_{n} t\end{cases}
$$



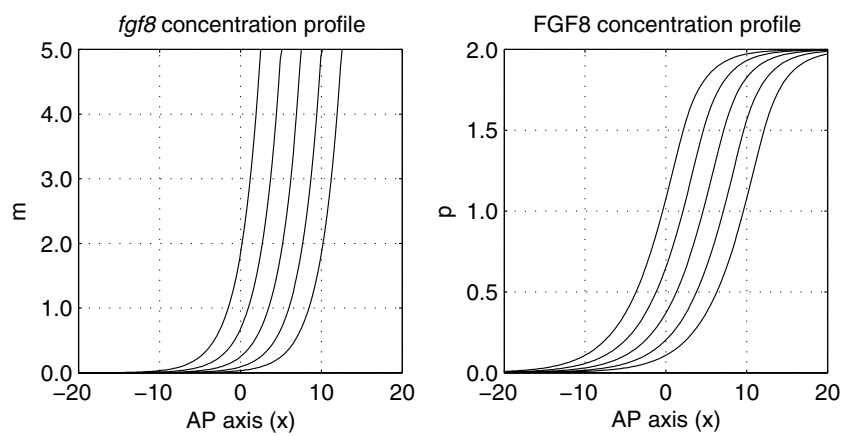

Fig. 4. Plots of the analytical solutions for the $f g f 8$ and FGF8 concentrations given by equations (26) and (29) respectively. Both plots show the wavefront over the series of time points $t=5,10,15,20,25$. The wave moves in a posterior direction (i.e. from left to right) as time progresses. Parameters are as follows: $\eta=0.5, D_{p}=10, \beta=0.2, c_{n}=0$ and $x_{n}=0.0$.

The above solution for $m$ is in travelling wave form since if $x-c_{n} t=X-c_{n} T$ then for $x>x_{n}+c_{n} t$ we have the trivial result $m(x, t)=m(X, T)$ and otherwise

$$
\begin{aligned}
m(x, t) & =\frac{1}{\beta} \exp \left[\beta\left(\frac{x-x_{n}}{c_{n}}-t\right)\right] \\
& =\frac{1}{\beta} \exp \left[\beta\left(\frac{x-x_{n}}{c_{n}}-\frac{x-X+c_{n} T}{c_{n}}\right)\right] \\
& =m(X, T) .
\end{aligned}
$$

Figure 4 shows a plot of the $f g f 8$ profile given by equation (26) over a series of time points.

\subsection{The protein profile}

Given the travelling wave nature of the mRNA profile in equation (26) we can solve equation (15) for the protein by transforming to travelling wave coordinates of the form $z=x-x_{n}-c_{n} t$; we have

$$
D_{p} p^{\prime \prime}+c_{n} p^{\prime}-\eta p= \begin{cases}-e^{-\frac{\beta z}{c_{n}}} & \text { if } \quad z \leq 0 \\ -1 & \text { if } \quad z>0\end{cases}
$$

where $^{\prime}=d / d z$. The above can be solved in each subinterval to give (assuming bounded solutions as $z \rightarrow \pm \infty$ and a differentiably continuous solution at $z=0$ ):

$$
p(x, t)=\left\{\begin{array}{lll}
A_{l} \exp \left\{n_{+}\left(x-x_{n}-c_{n} t\right)\right\}+A \exp \left\{\beta \frac{\left(x-x_{n}\right)}{c_{n}}\right\} & \text { if } & x-x_{n}-c_{n} t \leq 0, \\
B_{r} \exp \left\{n_{-}\left(x-x_{n}-c_{n} t\right)\right\}+\frac{1}{\eta} & \text { if } & x-x_{n}-c_{n} t>0,
\end{array}\right.
$$

where $A, n_{ \pm}, A_{l}$ and $B_{r}$ are as in equations (22) and (23). The FGF8 profile given by equation (29) is plotted in Figure 4. 


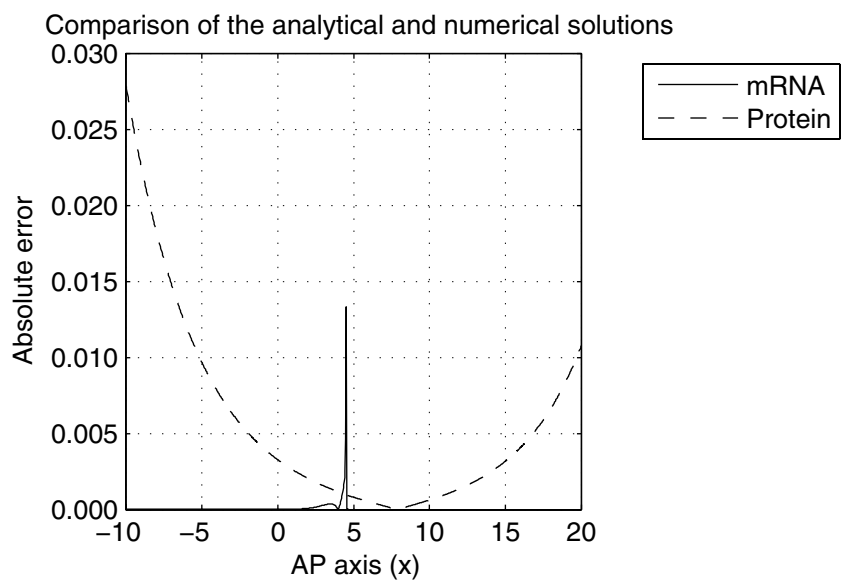

Fig. 5. Plots of the absolute error between the numerical and analytical solutions for the mRNA and protein profiles. The analytical profiles are given by equations (26) and (29) respectively. Parameters are as follows: $\eta=1.0, D_{p}=20, \beta=1.0, c_{n}=0.5$ and $x_{n}=0.0$.

Figure 5 shows a plot of the absolute error between the analytical and numerical solutions for the mRNA and protein profiles. There is little error over the whole domain for the mRNA profile but the zero flux boundary conditions of the numerical solution result in a more noticeable discrepancy between the protein profiles near the boundaries of the domain.

\subsection{Approximations for the FGF8 dynamics}

If $f g f 8$ decay is very rapid there is little FGF8 synthesis outside of the tail region. In this case we can make the simplifying assumption that $f g f 8$ is only translated into FGF8 in the tail of the embryo. This would require only a single equation to model the concentration of FGF8 along the AP axis and make future analysis simpler. The likely effect of this assumption would be to make the FGF8 gradient steeper, but this is an effect that could be overcome by adjusting the rate at which FGF8 diffuses. At present there is little experimental evidence on the magnitude of any parameter used in this model and the FGF8 dynamics are coupled into the signalling model purely as a readout. Therefore we feel that this simplification is reasonable.

Supposing that translation of FGF8 is limited to the tail region of the embryo; then instead of the pair of equations describing $f g f 8$ and FGF8, we can consider a single equation for the dynamics of FGF8. In non-dimensional form, this equation is:

$$
\frac{\partial w}{\partial t}=\chi_{w}-\eta w+D_{w} \frac{\partial^{2} w}{\partial x^{2}},
$$

where $w$ is the FGF8 concentration (with protein synthesis limited to the tail region) and $\chi_{w}=H\left(x-x_{n}-c_{n} t\right)$. The boundary conditions for $w$ are given by

$$
\begin{aligned}
& w \text { is bounded as } x-\left\{x_{n}+c_{n} t\right\} \rightarrow+\infty, \\
& w \rightarrow 0 \text { as } x-\left\{x_{n}+c_{n} t\right\} \rightarrow-\infty
\end{aligned}
$$


The above equation can be solved in travelling wave coordinates, of the form $z=x-x_{n}-c_{n} t$, to give

$$
w(x, t)=\left\{\begin{array}{cl}
\frac{n_{-}}{\eta\left(n_{-}-n_{+}\right)} \exp \left\{n_{+}\left(x-x_{n}-c_{n} t\right)\right\} & \text { if } \quad x-x_{n}-c_{n} t \leq 0, \\
\frac{\left.n_{+} n_{+}\right)}{\eta\left(n_{-}-n_{+}\right.} \exp \left\{n_{-}\left(x-x_{n}-c_{n} t\right)\right\}+\frac{1}{\eta} & \text { if } \quad x-x_{n}-c_{n} t>0,
\end{array}\right.
$$

where $n_{ \pm}$are as defined in equation (22) but with $D_{p}=D_{w}$.

Figure 6 compares the protein profile with and without local translation of FGF8 for a range of values of $\beta$, the rate of mRNA decay. When $\beta$ is small, there is a noticeable slackening in the FGF8 gradient as more $f g f 8$ remains to translate FGF8. As $\beta$ is increased the FGF8 gradient tends to the limiting case in which there is no local translation.

Currently there is limited experimental data to suggest which parameter regime should be chosen and hence which of the model systems (including and excluding fgf8 mRNA dynamics) is most accurate. However, since both of the models eventually give rise to travelling waves in FGF8 we choose to investigate the effects of FGF8 perturbation in the regime in which FGF8 translation is limited to the tail.

\section{Reduced model}

As a result of the assumptions made in the previous section, we choose to model somite formation using the following non-dimensional model:

$$
\frac{\partial u}{\partial t}=\frac{(u+\mu v)^{2}}{\gamma+u^{2}} \chi_{u}-u
$$

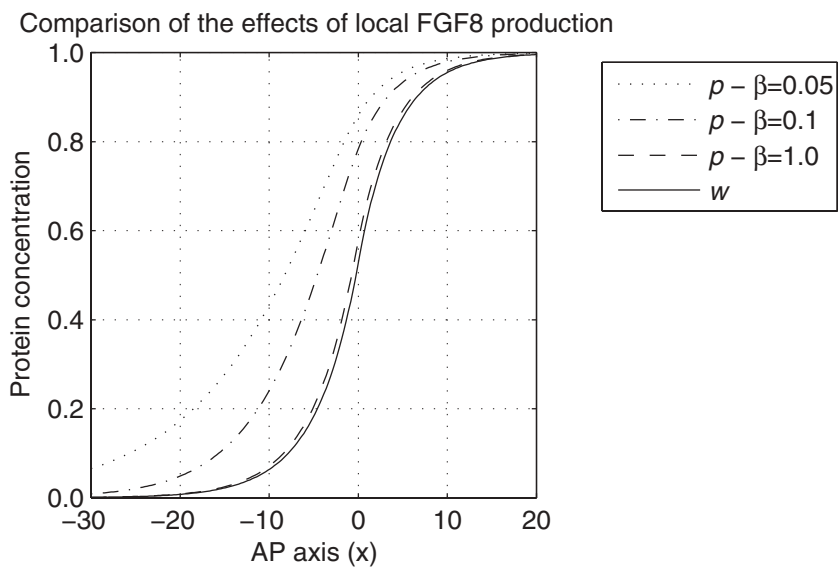

Fig. 6. The changes in the protein concentration profile $(p)$ as $\beta$, the linear decay rate of $f g f 8$, is varied. The dotted, dash-dotted and dashed lines show plots of the gradient given by equation (29) for $\beta=0.05,0.1,1.0$ respectively and the solid profile shows the gradient when protein production is restricted to the tail region, given by equation (32). As $\beta$ is increased towards 1.0 the $p$ gradient becomes more similar to the $w$ gradient, as expected. Parameters are as follows: $\eta=1.0, D_{p}=D_{w}=20, x_{n}=0.0, t=0.0$ and $c_{n}=0$. 


$$
\begin{aligned}
\frac{\partial v}{\partial t} & =\kappa\left(\frac{\chi_{v}}{\epsilon+u}-v\right)+D_{v} \frac{\partial^{2} v}{\partial x^{2}} \\
\frac{\partial w}{\partial t} & =\chi_{w}-\eta w+D_{w} \frac{\partial^{2} w}{\partial x^{2}} .
\end{aligned}
$$

As in the previous section, $u$ represents the concentration of somitic factor, $v$ the concentration of signalling molecule and $w$ the concentration of FGF8. Production of $u, v$ and $w$ are controlled by the respective Heaviside functions

$$
\begin{aligned}
& \chi_{u}=H\left(w^{*}-w\right), \\
& \chi_{v}=H\left(t-t^{*}\left(w^{*}, x\right)-t_{s}\right), \\
& \chi_{w}=H\left(x-x_{n}-c_{n} t\right),
\end{aligned}
$$

where $w^{*}$ is the level of FGF8 at the determination front, $t^{*}\left(w^{*}, x\right)$ is the time at which a cell at $x$ reaches the determination front (i.e. $\left.w\left(x, t^{*}\right)=w^{*}\right), t_{s}$ is the period of the segmentation clock, $x_{n}$ represents the initial position of the tail and $c_{n}$ represents the rate at which the AP axis is extending.

\subsection{Boundary conditions}

The boundary conditions are taken to be as in the previous models:

$$
\begin{aligned}
& u, v \rightarrow 0 \text { as } x-\left\{x_{n}+c_{n} t\right\} \rightarrow+\infty, \\
& u, v \text { are bounded as } x-\left\{x_{n}+c_{n} t\right\} \rightarrow-\infty, \\
& w \text { are bounded as } x-\left\{x_{n}+c_{n} t\right\} \rightarrow+\infty, \\
& w \rightarrow 0 \text { as } x-\left\{x_{n}+c_{n} t\right\} \rightarrow-\infty
\end{aligned}
$$

\subsection{Initial conditions}

The initial conditions for $u$ and $v$ are given by equations (17) and (18) and the initial condition for $w$ is taken to be the state of the travelling wave at time $t=0$ :

$$
w_{0}(x)=\left\{\begin{array}{lll}
\frac{n_{-}}{\eta\left(n_{-}-n_{+}\right)} \exp \left\{n_{+}\left(x-x_{n}\right)\right\} & \text { if } & x-x_{n} \leq 0, \\
\frac{n_{+}}{\eta\left(n_{-}-n_{+}\right)} \exp \left\{n_{-}\left(x-x_{n}\right)\right\}+\frac{1}{\eta} & \text { if } & x-x_{n}>0 .
\end{array}\right.
$$

\subsection{Graphical solution of the reduced model}

Given the travelling wave solution for the FGF8 profile, equation (32), we see that the path of the determination front, $w(x, t)=w^{*}$, is a straight line of the form $x=c_{n} t+\alpha$, where $\alpha$ is a constant determined by the value of $w^{*}$. Knowing the path of the determination front enables us to plot the paths of the discontinuities of the Heaviside functions $\chi_{u}$ and $\chi_{v}$ and further, to find the positions of the somite boundaries.

Figure 7(a) shows a plot of the pattern of somites formed in a control embryo using the reduced model. The discontinuities in $\chi_{u}$ and $\chi_{v}$ move along the AP at constant speed resulting in the formation of a segmentation pattern which is tightly regulated both spatially and temporally. 


\subsection{Numerical solution of the reduced model}

As with the full model, we solved the reduced model numerically using the NAG library routine D03PCF. Taking $t_{s}=1 / c_{n}$ so that somites are of approximately unit length, we have

$$
w^{*}=\frac{n_{-}}{\eta\left(n_{-}-n_{+}\right)} e^{n_{+}\left(-x_{n}+c_{n} t_{s}\right)}=\frac{n_{-}}{\eta\left(n_{-}-n_{+}\right)} e^{n_{+}\left(-x_{n}+1\right)} .
$$

Figure 8 shows the numerical solution of the model for a control embryo. The figure shows a sequence of coherent jumps in the somitic factor as a result of a set of periodic pulses in the signalling molecule. Both the graphical and numerical solutions are in agreement with experimental observations.

\section{Local application of FGF8}

We now consider adapting the model with the aim of mimicking experiments in which heparin beads soaked in FGF8 are implanted alongside the PSM. Results from such experiments carried out by Pourquié and co-workers [5] show the formation of a sequence of anomalous somites surrounding the bead: a series of up to 6-7 smaller somites forms anterior to the bead and a large somite forms posterior to the bead.

We modify the model by assuming that there is another source of FGF8 in the PSM which remains at a fixed axial level throughout somite formation and releases FGF8 into the surrounding tissue. The modified non-dimensional equations are

$$
\frac{\partial u}{\partial t}=\frac{(u+\mu v)^{2}}{\gamma+u^{2}} \chi_{u}-u,
$$
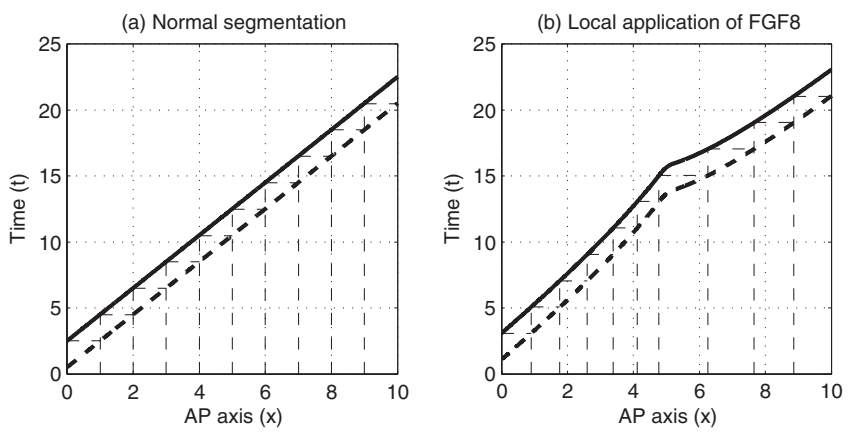

Fig. 7. The pattern of somites formed by the reduced model in (a) a normal embryo (see Section 4.3) and (b) with local application of FGF8 (see equation (58)). The bold, dashed line depicts the Heaviside function for $u$ and therefore the time at which cells reach the determination front and become able to produce somitic factor. The bold, solid line depicts the Heaviside function for $v$ and shows the time at which cells become able to send out a signal. The boundaries of the presumptive somites are marked by the thin dashed lines. The positions of the somite boundaries are found by tracing between the two lines. Parameters are as follows: $\eta=1.0, D_{w}=10, x_{n}=0.0, c_{n}=0.5, x_{b}=5.0, \phi=3.0, \xi=0.2$ and $w^{*}=0.5$. 
(a) Somitic factor (u)

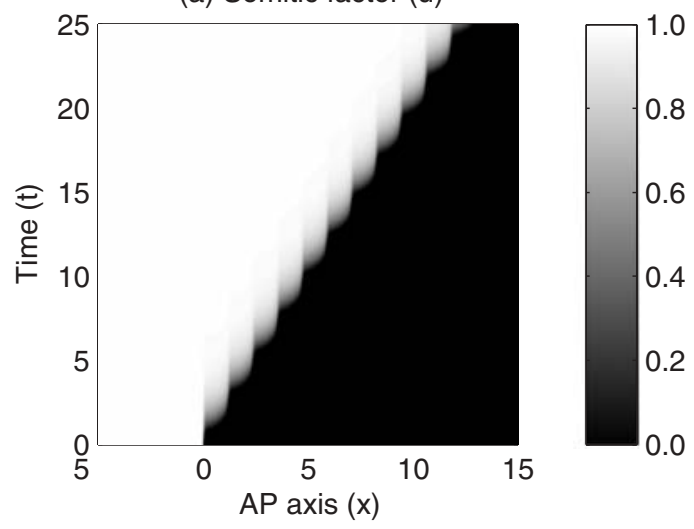

(b) Signal (v)

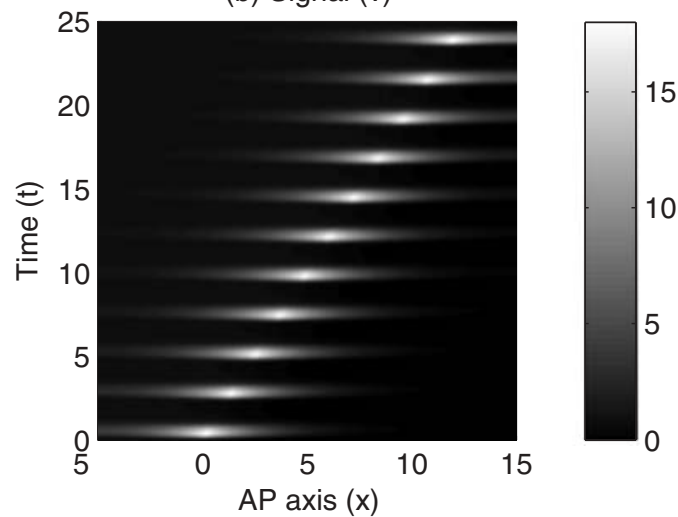

(c) FGF8 (w)

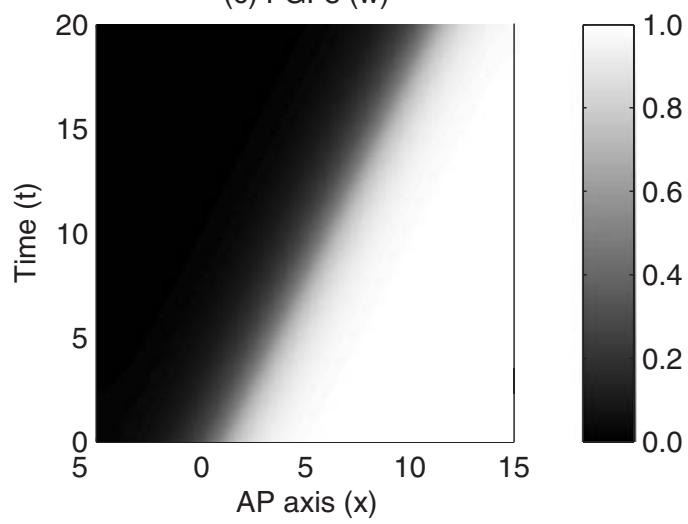

Fig. 8. Numerical solution of the new mathematical formulation of the Clock and Wavefront model for somite formation, given by equations (33)-(35), showing the spatio-temporal dynamics of the somitic factor (a), the signalling molecule, (b), and FGF8, (c). Parameters are as follows: $\mu=10^{-4}, \gamma=10^{-3}, \kappa=10, \epsilon=10^{-3}, \epsilon_{1}=10^{-3}, \eta=1.0, \phi=0.0$, $D_{v}=50, D_{w}=20, x_{n}=0.0, c_{n}=0.5, x_{b}=5.0, \xi=0.5$. 


$$
\begin{aligned}
& \frac{\partial v}{\partial t}=\kappa\left(\frac{\chi_{v}}{\epsilon+u}-v\right)+D_{v} \frac{\partial^{2} v}{\partial x^{2}}, \\
& \frac{\partial w}{\partial t}=\chi_{w}+\phi \chi_{b}-\eta w+D_{w} \frac{\partial^{2} w}{\partial x^{2}},
\end{aligned}
$$

where $\chi_{u}, \chi_{v}$ and $\chi_{w}$ are given by equations (36), (37) and (38) respectively, and $\chi_{b}=H\left(\xi-x_{b}+x\right) H\left(\xi+x_{b}-x\right)$ represents a source of FGF8 from a bead implant. $\phi$ is a measure of the strength of the bead source relative to the source in the tail, $x_{b}$ is the position of the midpoint of the bead and $\xi$ is a measure of the width of the bead ( $\chi_{b}$ is non-zero over a region of width $2 \xi$, centered at $x_{b}$ ). We assume the same initial and boundary conditions for $u, v$ and $w$ as in the control model. Note that this assumes that the bead is implanted at time $t=0$ : initially there is no effect on the endogenous gradient of FGF8 but as times increases, diffusion of FGF8 from the bead has an effect on the gradient which evolves as the somites are forming.

\subsection{Numerical solution}

We solved the model with local application of FGF8 given by equations (42)-(44) using the NAG library routine D03PCF as in the control case. Figure 9 shows the numerical solution of the model: with local application of FGF8, a sequence of smaller somites forms ahead of the bead implant and a large somite forms behind the bead, as predicted using the approximate model and seen in vivo.

\subsection{Solution of the FGF8 equation}

Equation (44) can be solved by finding $w_{e}$ and $w_{b}$ such that $w_{e}$ solves the equation for the endogenous gradient of FGF8:

$$
\frac{\partial w_{e}}{\partial t}=\chi_{w}-\eta w_{e}+D_{w} \frac{\partial^{2} w_{e}}{\partial x^{2}}
$$

with boundary conditions

$$
\begin{aligned}
& w_{e} \text { is bounded as } x_{n}-c_{n} t \rightarrow+\infty \\
& w_{e} \rightarrow 0 \text { as } x_{n}-c_{n} t \rightarrow-\infty
\end{aligned}
$$

and initial conditions given by $w_{e}(x, 0)=w_{0}(x)$, and such that $w_{b}$ solves the equation for the bead gradient of FGF8:

$$
\frac{\partial w_{b}}{\partial t}=\phi \chi_{b}-\eta w_{b}+D_{w} \frac{\partial^{2} w_{b}}{\partial x^{2}},
$$

with boundary conditions

$$
w_{b} \rightarrow 0 \quad \text { as } \quad x_{n}-c_{n} t \rightarrow \pm \infty,
$$

and initial conditions given by $w_{b}(x, 0)=0$. Then $w=w_{e}+w_{b}$ solves (44). 
(a) Somitic factor $(u)$

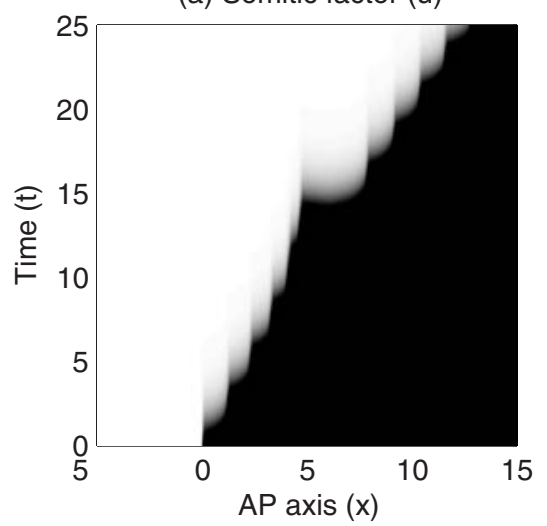

(b) Signal (v)

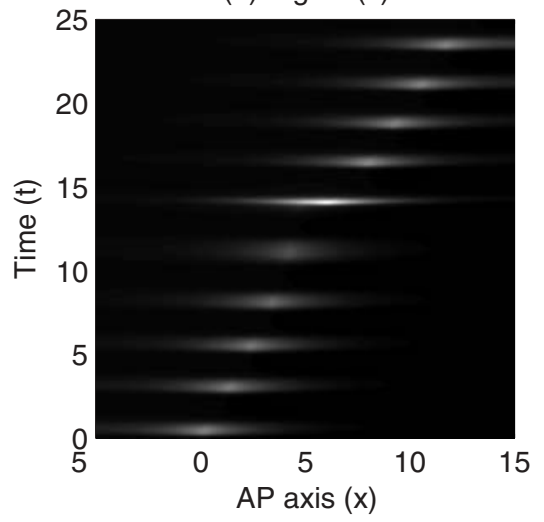

(c) FGF8 (w)

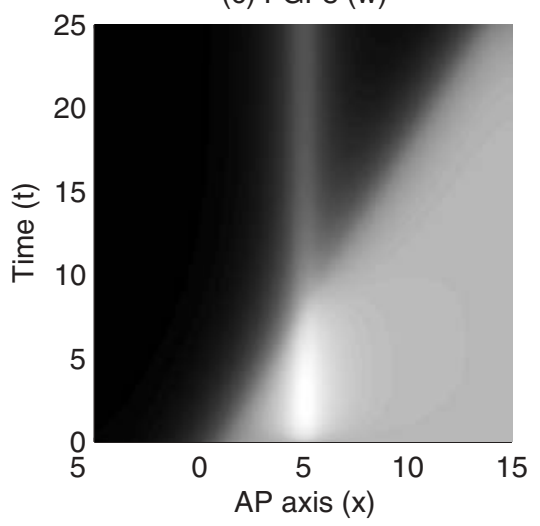

1.0

0.8

0.6

0.4

0.2

0.0

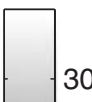

20

10

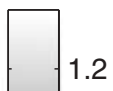

0.8

0.4

0.0

Fig. 9. Numerical solution of the new Clock and Wavefront model for somite formation, given by equations (42)-(44), showing the spatio-temporal dynamics of the somitic factor (a), the signalling molecule, (b), and FGF8, (c). With a source of FGF8 implanted in the PSM the somite anomalies are obvious. Parameters are as follows: $\mu=10^{-4}, \gamma=10^{-3}$, $\kappa=10, \epsilon=10^{-3}, \epsilon_{1}=10^{-3}, \eta=1.0, \phi=1.5, D_{v}=50, D_{w}=20, x_{n}=0.0, c_{n}=0.5$, $x_{b}=5.0, \xi=0.5$. 
Using the method of Fourier Transforms, equation (47) can be solved to give

$$
w_{b}(x, t)=\frac{\phi}{2 \eta} \int_{0}^{\eta t}\left[\operatorname{erf}\left(\frac{x-x_{b}+\xi}{\sqrt{4 D_{w} s / \eta}}\right)-\operatorname{erf}\left(\frac{x-x_{b}-\xi}{\sqrt{4 D_{w} s / \eta}}\right)\right] e^{-s} \mathrm{~d} s .
$$

In Figure 10, we plot the FGF8 profile set up by the bead implant as determined by equation (49).

\subsection{Approximation for the bead implant}

Figure 10 suggests that within the time taken to form one somite, the contribution to the FGF8 profile from a bead source reaches an approximately steady state. Letting $A_{ \pm}=\left(x-x_{b} \pm \xi\right) / \sqrt{4 D_{w} / \eta}$ we have

$$
\begin{aligned}
w_{b}(x, t)= & \frac{\phi}{\eta \sqrt{\pi}} \int_{0}^{\eta t}\left\{\int_{0}^{A_{+} / \sqrt{y}} e^{-v^{2}} \mathrm{~d} v-\int_{0}^{A_{-} / \sqrt{y}} e^{-v^{2}} \mathrm{~d} v\right\} \mathrm{d} y, \\
= & \frac{\phi}{\eta \sqrt{\pi}} \int_{0}^{\eta t}\left\{\operatorname{sign}\left(A_{+}\right) \int_{0}^{\left|A_{+}\right| / \sqrt{y}} e^{-v^{2}} \mathrm{~d} v\right. \\
& \left.-\operatorname{sign}\left(A_{-}\right) \int_{0}^{\left|A_{-}\right| / \sqrt{y}} e^{-v^{2}} \mathrm{~d} v\right\} e^{-y} \mathrm{~d} y .
\end{aligned}
$$

Interchanging the order of integration gives

$$
w_{b}(x, t)=\frac{\phi}{\eta \sqrt{\pi}} \operatorname{sign}\left(A_{+}\right) \int_{\left|A_{+}\right| / \sqrt{\eta t}}^{\infty} \int_{0}^{A_{+}^{2} / v^{2}} e^{-\left(y+v^{2}\right)} \mathrm{d} y \mathrm{~d} v
$$

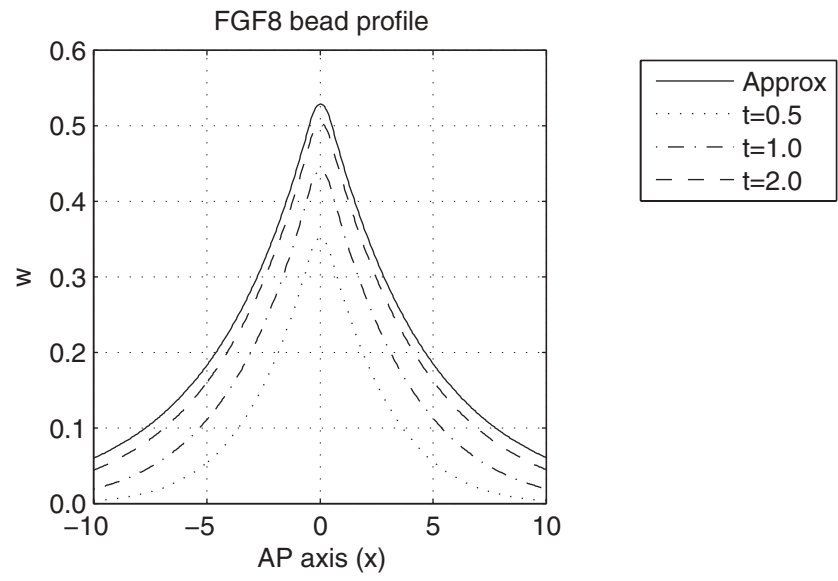

Fig. 10. Comparison between the analytical solution for the bead profile given by equation (47) and its approximation (54) as $t$ increases. The analytical solutions for $t=0.5,1.0,2.0$ are indicated by the dashed lines (see key for more details) and the approximate solution by the solid black line. We see that the analytical profile quickly tends to the steady state profile. Parameters are as follows: $\eta=1.0, D_{w}=20, x_{b}=0.0, \phi=5.0$ and $\xi=0.5$. 


$$
\begin{aligned}
& -\frac{\phi}{\eta \sqrt{\pi}} \operatorname{sign}\left(A_{-}\right) \int_{\left|A_{-}\right| / \sqrt{\eta t}}^{\infty} \int_{0}^{A_{-}^{2} / v^{2}} e^{-\left(y+v^{2}\right)} \mathrm{d} y \mathrm{~d} v, \\
= & \frac{\phi}{\eta \sqrt{\pi}} \operatorname{sign}\left(A_{+}\right) \int_{\left|A_{+}\right| / \sqrt{\eta t}}^{\infty} e^{-v^{2}}\left[1-e^{\left.-A_{+}^{2} / v^{2}\right]} \mathrm{d} v\right. \\
& -\frac{\phi}{\eta \sqrt{\pi}} \operatorname{sign}\left(A_{-}\right) \int_{\left|A_{-}\right| / \sqrt{\eta t}}^{\infty} e^{-v^{2}}\left[1-e^{-A_{-}^{2} / v^{2}}\right] \mathrm{d} v, \\
= & \frac{\phi}{2 \eta} \operatorname{sign}\left(A_{+}\right) \operatorname{erfc}\left(\frac{\left|A_{+}\right|}{\sqrt{\eta t}}\right)-\frac{\phi}{2 \eta} \operatorname{sign}\left(A_{-}\right) \operatorname{erfc}\left(\frac{\left|A_{-}\right|}{\sqrt{\eta t}}\right) \\
& -\frac{\phi}{\eta \sqrt{\pi}} \operatorname{sign}\left(A_{+}\right) \int_{\left|A_{+}\right| / \sqrt{\eta t}}^{\infty} e^{-v^{2}-A_{+}^{2} / v^{2}} \mathrm{~d} v \\
& +\frac{\phi}{\eta \sqrt{\pi}} \operatorname{sign}\left(A_{-}\right) \int_{\left|A_{-}\right| / \sqrt{\eta t}}^{\infty} e^{-v^{2}-A_{-}^{2} / v^{2}} \mathrm{~d} v .
\end{aligned}
$$

Consider

$$
\begin{aligned}
\int_{a}^{b} e^{-v^{2}-\frac{M^{2}}{v^{2}}} \mathrm{~d} v= & \frac{1}{2} \int_{a}^{b}\left(1+\frac{M}{v^{2}}\right) e^{-v^{2}-\frac{M^{2}}{v^{2}}} \mathrm{~d} v \\
& +\frac{1}{2} \int_{a}^{b}\left(1-\frac{M}{v^{2}}\right) e^{-v^{2}-\frac{M^{2}}{v^{2}}} \mathrm{~d} v
\end{aligned}
$$

This can be evaluated by making a change of variables: in the first integral let $u=v-M / v$ and in the second integral let $u=v+M / v$. Applying this to the previous equation,

$$
\begin{aligned}
w_{b}(x, t)= & \frac{\phi}{2 \eta} \operatorname{sign}\left(A_{+}\right) \operatorname{erfc}\left(\frac{\left|A_{+}\right|}{\sqrt{\eta t}}\right)-\frac{\phi}{2 \eta} \operatorname{sign}\left(A_{-}\right) \operatorname{erfc}\left(\frac{\left|A_{-}\right|}{\sqrt{\eta t}}\right) \\
& -\frac{\phi}{4 \eta} \operatorname{sign}\left(A_{+}\right)\left[1-\operatorname{erf}\left(\sqrt{\eta t}\left|A_{+}\right|\left\{\frac{A_{+}}{\eta}-t\right\}\right)\right] \exp \left(-2\left|A_{+}\right|\right) \\
& -\frac{\phi}{4 \eta} \operatorname{sign}\left(A_{+}\right)\left[1-\operatorname{erf}\left(\sqrt{\eta t}\left|A_{+}\right|\left\{\frac{A_{+}}{\eta}+t\right\}\right)\right] \exp \left(2\left|A_{+}\right|\right) \\
& +\frac{\phi}{4 \eta} \operatorname{sign}\left(A_{-}\right)\left[1-\operatorname{erf}\left(\sqrt{\eta t}\left|A_{-}\right|\left\{\frac{A_{-}}{\eta}-t\right\}\right)\right] \exp \left(-2\left|A_{-}\right|\right) \\
& +\frac{\phi}{4 \eta} \operatorname{sign}\left(A_{-}\right)\left[1-\operatorname{erf}\left(\sqrt{\eta t}\left|A_{-}\right|\left\{\frac{A_{-}}{\eta}+t\right\}\right)\right] \exp \left(2\left|A_{-}\right|\right) .
\end{aligned}
$$

Letting $t \rightarrow \infty$ gives the steady state solution for the bead implant:

$$
w_{b s}(x)=\left\{\begin{array}{c}
\frac{\phi}{2 \eta}\left[\exp \left(\frac{x-x_{b}+\xi}{\sqrt{D_{w} / \eta}}\right)-\exp \left(\frac{x-x_{b}-\xi}{\sqrt{D_{w} / \eta}}\right)\right] \\
\text { if } x \leq x_{b}-\xi, \\
\frac{\phi}{\eta}-\frac{\phi}{2 \eta}\left[\exp \left(\frac{x-x_{b}-\xi}{\sqrt{D_{w} / \eta}}\right)+\exp \left(-\frac{x-x_{b}+\xi}{\sqrt{D_{w} / \eta}}\right)\right] \\
\text { if } x_{b}-\xi<x \leq x_{b}+\xi, \\
\frac{\phi}{2 \eta}\left[\begin{array}{c}
\left.\exp \left(-\frac{x-x_{b}-\xi}{\sqrt{D_{w} / \eta}}\right)-\exp \left(-\frac{x-x_{b}+\xi}{\sqrt{D_{w} / \eta}}\right)\right] \\
\text { if } x>x_{b}+\xi .
\end{array}\right.
\end{array}\right.
$$


The steady state profile for the bead is also plotted in Figure 10. It can be seen that even after two time steps, the temporally varying profile is very close to the analytical approximation given by equation (54). We use this approximation in the following sections to find approximations for the paths of the discontinuities in the Heaviside functions defining $\chi_{u}$ and $\chi_{v}$ and hence to find the pattern of somites formed.

\subsection{Approximation for the FGF8 profile}

Using the steady state approximation for the bead profile given by equation (54) and the steady state travelling wave solution for the endogenous FGF8 profile given by equation $(32)$, the $(x, t)$ plane can be divided up into six regions to give

$$
w(x, t)=\left\{\begin{array}{r}
\frac{n_{-}}{\eta\left(n_{-}-n_{+}\right)} e^{n_{+} X}+\frac{\phi}{2 \eta}\left[e^{A_{+}}-e^{A_{-}}\right] \\
\text {if } x \leq x_{b}-\xi \quad \text { and } x \leq x_{n}+c_{n} t, \\
\frac{n_{+}}{\eta\left(n_{-}-n_{+}\right)} e^{n_{-} X}+\frac{1}{\eta}+\frac{\phi}{2 \eta}\left[e^{A_{+}}-e^{A_{-}}\right] \\
\text {if } x \leq x_{b}-\xi \quad \text { and } x>x_{n}+c_{n} t, \\
\frac{n_{-}}{\eta\left(n_{-}-n_{+}\right)} e^{n_{+} X}+\frac{\phi}{\eta}-\frac{\phi}{2 \eta}\left[e^{A_{-}}+e^{-A_{+}}\right] \\
\text {if } x_{b}-\xi<x \leq x_{b}+\xi \text { and } x \leq x_{n}+c_{n} t, \\
\frac{n_{+}}{\eta\left(n_{-}-n_{+}\right)} e^{n_{-} X}+\frac{1}{\eta}+\frac{\phi}{\eta}-\frac{\phi}{2 \eta}\left[e^{A_{-}}+e^{-A_{+}}\right] \\
\text {if } x_{b}-\xi<x \leq x_{b}+\xi \quad \text { and } x>x_{n}+c_{n} t, \\
\frac{n_{-}}{\eta\left(n_{-}-n_{+}\right)} e^{n_{+} X}+\frac{\phi}{2 \eta}\left[e^{\left.-A_{-}-e^{-A_{+}}\right]}\right. \\
\text {if } x>x_{b}+\xi \text { and } x \leq x_{n}+c_{n} t, \\
\frac{n_{+}}{\eta\left(n_{-}-n_{+}\right)} e^{n_{-} X}+\frac{1}{\eta}+\frac{\phi}{2 \eta}\left[e^{\left.-A_{-}-e^{-A_{+}}\right]}\right. \\
\text {if } x>x_{b}+\xi \text { and } x>x_{n}+c_{n} t,
\end{array}\right.
$$

where $n_{ \pm}$are given by equation (22),

$$
A_{ \pm}=\frac{x-x_{b} \pm \xi}{\sqrt{D_{w} \eta}} \quad \text { and } \quad X=x-x_{n}-c_{n} t
$$

To find the path of the determination front in the $(x, t)$ plane, we need to solve $w(x, t)=w^{*}$, where $w^{*}$ is the level of FGF8 expressed at the determination front. First we must test to find which branch of the travelling wave solution for the endogenous FGF8 profile is valid: we do this by imposing the test condition

If $w_{e}\left(x,\left(x-x_{n}\right) / c_{n}\right)\left\{\begin{array}{l}\geq w^{*} \text { then use the } t \geq \frac{x-x_{n}}{c_{n}} \quad \text { branch of the solution, } \\ <w^{*} \text { then use the } t<\frac{x-x_{n}}{c_{n}} \quad \text { branch of the solution, }\end{array}\right.$ 
where $w_{e}(x, t)$ is the endogenous FGF8 profile given by equation (32). The position of the determination front is therefore given by

$$
t=\left\{\begin{array}{l}
-\frac{1}{n_{+} c_{n}} \ln \left\{\frac{\eta\left(n_{-}-n_{+}\right)}{n_{-}} e^{n_{+}\left(x_{n}-x\right)}\left(w^{*}-\frac{\phi}{2 \eta}\left[e^{A_{+}}-e^{A_{-}}\right]\right)\right\} \\
\text {if } x \leq x_{b}-\xi \text { and } w_{e}\left(x,\left(x-x_{n}\right) / c_{n}\right) \geq w^{*}, \\
-\frac{1}{n_{-} c_{n}} \ln \left\{\frac{\eta\left(n_{-}-n_{+}\right)}{n_{+}} e^{n_{-}\left(x_{n}-x\right)}\left(w^{*}-\frac{1}{\eta}-\frac{\phi}{2 \eta}\left[e^{A_{+}}-e^{A_{-}}\right]\right)\right\} \\
\text {if } x \leq x_{b}-\xi \text { and } w_{e}\left(x,\left(x-x_{n}\right) / c_{n}\right)<w^{*}, \\
-\frac{1}{n_{+} c_{n}} \ln \left\{\frac{\eta\left(n_{-}-n_{+}\right)}{n_{-}} e^{n_{+}\left(x_{n}-x\right)}\left(w^{*}-\frac{\phi}{\eta}+\frac{\phi}{2 \eta}\left[e^{A-}+e^{-A_{+}}\right]\right)\right\} \\
\text {if } x_{b}-\xi<x \leq x_{b}+\xi \text { and } w_{e}\left(x,\left(x-x_{n}\right) / c_{n}\right) \geq w^{*}, \\
-\frac{1}{n_{-} c_{n}} \ln \left\{\frac{\eta\left(n_{-}-n_{+}\right)}{n_{+}} e^{n_{-}\left(x_{n}-x\right)}\left(w^{*}-\frac{1}{\eta}-\frac{\phi}{\eta}+\frac{\phi}{2 \eta}\left[e^{A-}+e^{-A_{+}}\right]\right)\right\} \\
\text {if } x_{b}-\xi<x \leq x_{b}+\xi \text { and } w_{e}\left(x,\left(x-x_{n}\right) / c_{n}\right)<w^{*}, \\
-\frac{1}{n_{+} c_{n}} \ln \left\{\frac{\eta\left(n_{-}-n_{+}\right)}{n_{-}} e^{n_{+}\left(x_{n}-x\right)}\left(w^{*}-\frac{\phi}{2 \eta}\left[e^{-A_{-}}-e^{-A_{+}}\right]\right)\right\} \\
\text {if } x>x_{b}+\xi \text { and } w_{e}\left(x,\left(x-x_{n}\right) / c_{n}\right) \geq w^{*}, \\
-\frac{1}{n_{-} c_{n}} \ln \left\{\frac{\eta\left(n_{-}-n_{+}\right)}{n_{+}} e^{n_{-}\left(x_{n}-x\right)}\left(w^{*}-\frac{1}{\eta}-\frac{\phi}{2 \eta}\left[e^{-A_{-}-}-e^{-A_{+}}\right]\right)\right\} \\
\text {if } x>x_{b}+\xi \text { and } w_{e}\left(x,\left(x-x_{n}\right) / c_{n}\right)<w^{*} .
\end{array}\right.
$$

As discussed earlier, the path of the determination front is a line in $(x, t)$ space. Figure 11 shows a series of plots of the progress of the determination front as key parameters for the bead implant are varied. As expected, increasing the strength of the source and the size of the implant causes the determination front to deviate further from its normal course. Conversely, if the level of FGF8 expressed at the determination front is decreased then the effects of a bead implant are more noticeable.

\subsection{Application to the model}

We can apply the above result to find the positions of the discontinuities in the Heaviside functions of equations (36) and (37) and therefore predict the pattern of somites formed. Figure 7(b) shows a plot of the $(x, t)$ phase plane in the perturbed case with the positions of the discontinuities in $\chi_{u}$ and $\chi_{v}$ and the somite boundaries clearly marked. The diagram shows a sequence of approximately four small somites anterior to the bead and a large somite posterior to the bead, as observed experimentally.

\section{Discussion}

In this paper we presented a mathematical formulation of Pourquié's Clock and Wavefront model for somite formation. We solved the decoupled equations for $f g f 8$ and FGF8 analytically and proceeded to make some simplifying assumptions to allow prediction of the somite anomalies formed when the wavefront is perturbed locally. We solved the simplified model numerically: the control case showed a pattern of somites formed in a manner that was tightly regulated, both spatially and temporally. The perturbed case showed a sequence of anomalous somites which 
(a) Varying $\phi$

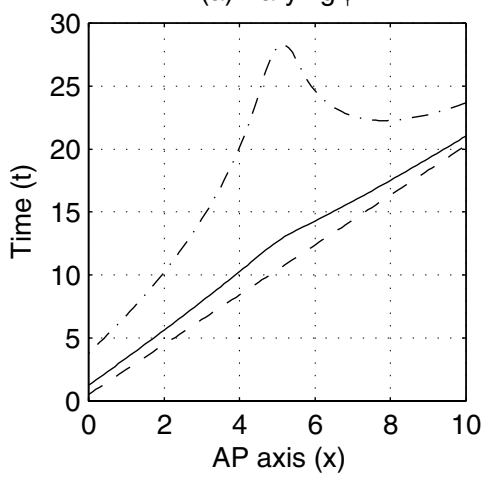

(b) Varying $\xi$

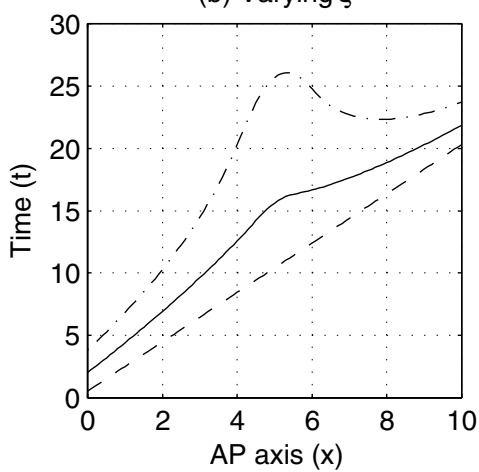

(c) Varying w*

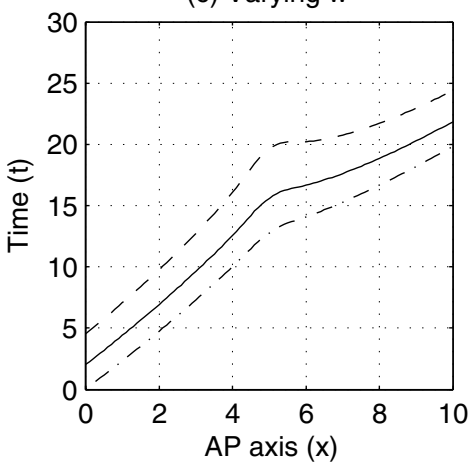

$$
\begin{array}{r}
--\phi=0.0 \\
-\cdot \phi=1.0 \\
-\phi=4.0
\end{array}
$$

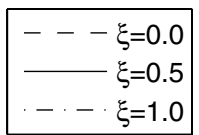

Fig. 11. The progress of the determination front, given by equation (58), along the AP axis as key parameters are varied. (a) Varying $\phi$, the relative strength of the bead source. (b) Varying $\xi$, the size of the bead implant. (c) Varying $w^{*}$, the level of FGF8 at the determination front. As $\phi$ and $\xi$ are increased and $w^{*}$ decreased the determination front is perturbed further from its usual path. Unless otherwise stated parameters are as follows: $\eta=1.0, D_{w}=20$, $x_{n}=0.0, c_{n}=0.5, x_{b}=5.0, \phi=1.0, \xi=0.5$ and $w^{*}=0.5$. 
mimicked those predicted by analysis of the model and also those seen in vivo [5]. There are a few points that it is important to note with regard to the model and we discuss them below.

Firstly we note that the model was based on three assumptions (see Section 2) and we use the results of Pourquié and co-workers [5] to justify the first and the third. The first is supported by the result that surgical inversions of the PSM result in somite anomalies only when the inverted tissue lies anterior to the determination front at the time of inversion, demonstrating that upon reaching the determination front cells make an irreversible commitment to a certain developmental pathway. Expression of certain genes in the PSM, such as Mesp2 [16,17], demonstrate this commitment and are candidates for a biological basis for the somitic factor. The third can be supported by noting that the effects of implantation of FGF8-soaked beads in the PSM are felt only up to the level of the determination front at the time of the implant, demonstrating that cells become refractory to FGF8 signalling once they have reached the determination front. However, at present, there is no biological basis for the signalling molecule of the second assumption: this is a hypothesis of the model based on the experimental work of Stern and co-workers [14, 15,20].

Secondly, we note that there is no quantitative data available on any of the parameters involved in the model. As a result of this we made some simplifying approximations that enabled us to get a greater analytical insight into the mechanisms of the model. The first assumption was to assume that mRNA decay is very rapid so that local mRNA translation of the protein can be effectively ignored. We note that both regimes (with and without local translation) result in qualitatively similar travelling wave profiles of FGF8 along the AP axis and the signalling basis uses a read out from these profiles in the form of a switch; therefore it seems reasonable to consider the reduced model for FGF8.

In assuming that the FGF8 profile from the bead reaches a steady state profile very quickly (in order to use the steady state approximation) we have implicitly assumed that diffusion of the protein is rapid. If that is not the case (as FGF8 protein is a relatively large molecule, this could be so), then the steady state approximation may not be accurate if the determination front is close to the bead at the time of implantation.

Thirdly, we note that it is likely that the effects of local application of FGF8 via a heparin-soaked bead are likely to wear off before all the PSM that would otherwise be affected by this perturbation has been gated into somites [13]. Decaying effects of local application of FGF8 could result in a pronounced large somite as the decay of the source would confer the potential to become somitic to many cells at the same time. A temporally varying local source of FGF8 is something that remains to be investigated.

Fourthly, we note that there could be some delay in FGF8 translation. Incorporation of a delay in the model can be easily achieved by alteration of the protein equation (15) so that the term involving $f g f 8$ is of the form $\beta m(t-\tau)$. Although a large delay in translation $(\tau)$ destroys the monotonicity of the gradient, we have investigated its effect and shown that the determination front will continue to move along the AP axis with constant speed, and at no point before reaching the determination front could a cell, however briefly, experience a level of FGF8 signalling 
below that expressed at the determination front. For this reason we do not consider incorporation of the delay any further.

Lastly, we note that the results of this paper clearly show the incorporation of certain cells into differently numbered somites than their control counterparts. It can be seen that such cells will go on to segment within a different time step and will therefore experience a different number of clock oscillations before segmenting. Dubrulle et al. [p. 220] [5] demonstrate that "FGF8 treatment can increase the number of clock oscillations experienced by PSM cells without altering their absolute axial position in tissue. Cells which experience an extra oscillation become incorporated into a differently numbered somite and exhibit Hox expression indicative of a more posterior fate when compared with contralateral control cells." Our model clearly accounts for this result.

It is very encouraging to see that our model can account for normal somite formation and mimic the results seen with local application of FGF8. In another paper [2] we use the models described here to make some experimentally testable hypotheses that we hope can be used to further verify the model.

Acknowledgements. REB would like to thank EPSRC for a Doctoral Training Award and Wadham College, Oxford for a Senior Scholarship. SS has been funded by the Research Training Fellowship programme in Mathematical Biology (Grant No. 069155) of the Wellcome Trust (London), and a Junior Fellowship of Christ Church (Oxford). PKM thanks the Biocomplexity Institute and the School of Informatics (Indiana University, Bloomington) for support and hospitality.

The authors would also like to express their kind thanks to Paul Kulesa and Olivier Pourquié for their kind hospitality at the Stowers Institute and to Olivier Pourquié once more for helpful comments on the manuscript.

\section{References}

1. Baker, R.E., Schnell, S., Maini, P.K.: Formation of vertebral precursors: past models and future predictions. J. Theor. Med. 5 (1), 23-35 (2003)

2. Baker, R.E., Schnell, S., Maini, P.K.: A clock and wavefront mechanism for somite formation. Submitted to Dev. Biol, 2005

3. Collier, J.R., McInerney, D., Schnell, S., Maini, P.K., Gavaghan, D.J., Houston, P., Stern, C.D.: A cell cycle model for somitogenesis: mathematical formulation and numerical solution. J. Theor. Biol. 207, 305-316 (2000)

4. Cooke J., Zeeman, E.C.: A clock and wavefront model for control of the number of repeated structures during animal morphogenesis. J. Theor. Biol. 58, 455-476 (1976)

5. Dubrulle, J., McGrew, M.J., Pourquié O.: FGF signalling controls somite boundary position and regulates segmentation clock control of spatiotemporal Hox gene activation. Cell 106, 219-232 (2001)

6. Dubrulle, J., Pourquié, O.: From head to tail: links between the segmentation clock and antero-posterior patterning of the embryo. Curr. Op. Gen. Dev. 5, 519-523 (2002)

7. Dubrulle, J., Pourquié, O.: $f g f 8$ mRNA decay establishes a gradient that couples axial elongation to pattering in the vertebrate embryo. Nature 427, 419-422 (2004)

8. Gossler, A., Hrabě de Angelis, M.: Somitogenesis. Curr. Top. Dev. Biol. 38, 225-287 (1998)

9. McInerney, D., Schnell, S., Baker, R.E., Maini, P.K.: A mathematical formulation for the cell cycle model in somitogenesis: parameter constraints and numerical solutions. IMA J. Math. Appl. Med. Biol. 21, 85-113 (2004) 
10. Palmeirim, I., Henrique, D., Ish-Horowicz, D., Pourquié, O.: Avian hairy gene expression identifies a molecular clock linked to vertebrate segmentation and somitogenesis. Cell 91, 639-648 (1997)

11. Pourquié, O.: The segmentation clock: converting embryonic time into spatial pattern. Science 301, 328-330 (2003)

12. Pourquié, O.: The chick embryo: a leading model for model in somitogenesis studies. Mech. Dev. 121, 1069-1079 (2004)

13. Pourquié, O.: Pers. Comm., 2004

14. Primmett, D.R.N., Norris, W.E., Carlson, G.J., Keynes, R.J., Stern, C.D.: Periodic anomalies induced by heat shock in the chick embryo are associated with the cell cycle. Development 105, 119-130 (1989)

15. Primmett, D.R.N., Stern, C.D., Keynes, R.J.: Heat shock causes repeated segmental anomalies in the chick embryo. Development 104, 331-339 (1988)

16. Saga, Y.: Genetic rescue of segmentation defect in MesP2-deficient mice by MesP1 gene replacement. Mech. Dev. 75, 53-66 (1998)

17. Saga, Y., Hata, N., Koseki, H., Taketo, M.M.: Mesp2: a novel mouse gene expressed in the presegmented mesoderm and essential for segmentation initiation. Genes Dev. 2, 835-845 (2001)

18. Schnell, S., Maini, P.K.: Clock and induction model for somitogenesis. Dev. Dyn. 217, 415-420 (2000)

19. Schnell, S., Maini, P.K., McInerney, D., Gavaghan, D.J., Houston, P.: Models for pattern formation in somitogenesis: a marriage of cellular and molecular biology. C.R. Biologies 325, 179-189 (2002)

20. Stern, C.D., Fraser, S.E., Keynes, R.J., Primmett, D.R.N.: A cell lineage analysis of segmentation in the chick embryo. Development 104S, 231-244 (1988)

21. Stickney, H.L., Barresi, M.S.J., Devoto, S.H.: Somite development in zebrafish. Dev. Dyn. 219, 287-303 (2000)

22. Stockdale, F.E., Nikovits, W.J., Christ, B.: Molecular and cellular biology of avian somite development. Dev. Dyn. 219, 304-321 (2000)

23. Tabin, C.J., Johnson, R.L.: Clocks and Hox. Nature 412, 780-781 (2001) 